\title{
Pseudogaps and Extrinsic Losses in Photoemission Experiments on Poorly Conducting Solids
}

\author{
Robert Joynt \\ Department of Physics \\ University of Wisconsin-Madison \\ 1150 University Avenue \\ Madison, WI 53706
}

(July 7, 2018)

\begin{abstract}
A photoelectron, on being emitted from a conducting solid, may suffer a substantial energy change through ohmic losses that can drastically alter the lineshape on the meV scale which is now observable due to improved resolution. Almost all of this energy loss takes place after the electron leaves the solid. These losses are expected to be important in isotropic materials with relatively low conductivity, such as certain colossal magnetoresistance manganates and very electrically anisotropic materials, such as one-dimensional conductors. Such effects may also be important in the interpretation of photemission in high- $\mathrm{T}_{c}$ superconductors. In all these materials, the electric field of the photoelectron can penetrate the system. In particular, extrinsic losses of this type can mimic pseudogap effects and other peculiar features of photoemission in cubic manganates. This is illustrated with the case of $\mathrm{La}_{0.67} \mathrm{Ca}_{0.33} \mathrm{MnO}_{3}$.
\end{abstract}


In the past few years, the resolution of photoemission (PE) experiments has improved to the range of $10 \mathrm{meV}$ or less, and this has allowed finer details of electronic structure to be observed, including the "pseudogap" - a depression of intensity at the chemical potential $\mu$. Pseudogaps have been observed in a wide variety of materials: quasi-one-dimensional (1D) systems, both inorganic ( $\left.\mathrm{Ta}_{\mathrm{Se}}\right)_{2} \mathrm{I}$ [1] and organic (TTF-TCNQ) [2], quasi-2D systems such as the underdoped high- $\mathrm{T}_{c}$ materials [3], and most recently 3D systems: the colossal magnetoresistance (CMR) manganates [4], [5]. In many cases, interesting temperature dependences of these pseudogaps have been observed. The origin of pseudogaps is among the most fundamental problems of present-day condensed matter physics. Because the most direct way to see them is with PE, it is well to understand this measurement very thoroughly.

A somewhat disturbing aspect of the current situation is that, although the resolution has greatly improved, isolated resolution-limited peaks are not the rule in angle-resolved photoemission (ARPES) data that detect pseudogaps. There is a suggestion here that some extrinsic broadening mechanism is at work or that a large unexplained background is present [6.

The conventional wisdom interpretation of ARPES data is that at a given wavevector $\vec{k}$, the ideal intensity $I(\omega)$ is proportional to $A(\vec{k}, \omega)$, the spectral function for a single hole. The observed intensity, at least near $\mu$, is broadened only because of the finite instrumental resolution. $A(\vec{k}, \omega)$ is, in this context, an "intrinsic" quantity. The outgoing electron either suffers a large energy loss due, for example, to plasmon emission or ionization, or suffers no loss. In the former case, the electron is not detected or its energy is sufficiently far from threshold that it is ignored; in the latter case, the electron is detected and its measured distribution is a faithful reflection of the intrinsic distribution in the solid.

This conventional picture of the photoemission process is reconsidered in this report for certain important classes of materials, namely those which are 'poor conductors'. The working definition of this phrase is a DC resistivity $\rho_{0}$ which exceeds the Mott value $\sim$ $100 \mu \Omega$-cm. I will argue that electrons emitted from such materials are subject to losses of the order of a few tens of meV after they leave the surface. At low resolutions, these 
processes are usually not important, but for high-resolution experiments they cannot be ignored.

If an electron is emitted normally at speed $v$ from very near a clean surface and leaves the sample without undergoing significant energy loss, then the Fourier transform of the electric field inside the material is

$$
\vec{E}(\vec{r}, \omega)=\frac{-e}{2 \pi v} \frac{2}{1+\epsilon(\omega)} \int_{0}^{\infty} d z^{\prime} e^{-i \omega z^{\prime} / v} \frac{\vec{r}-z^{\prime} \hat{z}}{\left|\vec{r}-z^{\prime} \hat{z}\right|^{3}}
$$

The surface is the $x-y$ plane, $-e$ is the charge on the electron, and $\epsilon(\omega)$ is the bulk dielectric function. This electric field can set up currents in the bulk.

To arrive at this expression certain approximations have been made. The expression for $\vec{E}$ does not hold when the charge is within a few atomic layers of the surface: to model the short-time, high-frequency losses, a proper treatment using the surface dielectric function would be required. I do not attempt this here, as only the low-frequency loss is of interest. I assume the normal skin effect - the wavevector dependence of $\epsilon(\omega)$ has been neglected. At high frequencies or for very low temperatures for clean systems, the anomalous skin effect should be taken into account. The factor $2 /(1+\epsilon)$ in Eq. 1 gives image charge and screening effects and proves critical.

These formulas depend on the assumption that the material is cubic. The important special case of emission along the z-axis of a tetragonal material may be treated by the same method, and the image charge factor becomes $2 /\left(1+\sqrt{\epsilon_{x x} \epsilon_{z z}}\right)$. Thus the absorption is strongly enhanced in a layered conducting material where we expect $\left|\epsilon_{x x}\right|>>\left|\epsilon_{z z}\right|$ at the relevant frequencies. Similar remarks apply to the orthorhombic 1D conductor case, but the calculations become far more complicated and no simple expression comparable to Eq. 1 could be derived.

The currents set in motion by the field will produce ohmic loss. These will be represented in the observed energy of the electron. Classically, the total energy loss is given by

$$
Q=\frac{1}{2} \int_{-\infty}^{\infty} d \omega \int d^{3} r \Re \sigma(\omega)|\vec{E}(\vec{r}, \omega)|^{2}=\frac{2 e^{2}}{\pi v} \mathcal{C} \int_{0}^{\infty} d \omega \frac{L(\omega)}{\omega}
$$


where $\mathcal{C} \approx 2.57$ and $L(\omega)=\Re \sigma(\omega) /|1+\epsilon(\omega)|^{2}$.

This classical calculation corresponds to a quantum-mechanical one. In fact, as constant electron velocity was assumed, it is the Born approximation. Because the field is appropriately screened by the dielectric function, I term it the screened Born approximation. This approximation should be valid for those electrons whose energy loss is small compared with their total energy. This ratio is of order $50 \mathrm{mev} / 20 \mathrm{eV} \sim 2.5 \times 10^{-3}$ for experimental parameters of interest. The relative differential probability is obtained by setting

$$
Q=\hbar \int_{0}^{\infty} \omega P(\omega) d \omega
$$

where $P(\omega)$ is the relative differential probability of losing energy $\hbar \omega$. Hence

$$
P(\omega)=\frac{2 e^{2} \mathcal{C} L(\omega)}{\pi \hbar v \omega^{2}}
$$

This expression is general, and is of course related to well-known formulas in electronenergy-loss spectroscopy [7]. Its relevance to PE has been noted before [8]. Refs. [0] and 8] are concerned with plasmon and other losses in the electron volt range. Recent work on processes occurring when the electron is still inside the material has also clarified the losses in this energy range [6], while highlighting the lack of explanation of background in the millielectron volt range.

Note at this point that $P(\omega)$ at low frequencies is greater for systems with low conductivity. Because $\epsilon(\omega) \sim 4 \pi i \sigma / \omega$ we have $P(\omega) \sim \sigma / \omega^{2}|\epsilon|^{2} \sim 1 / \sigma$.

Quantum mechanics requires some probability for forward scattering $P_{0}$, or that the electron loses zero energy. Thus, the total normalization is given by the equation

$$
1=P_{0}+\int_{0}^{\infty} P(\omega) d \omega
$$

$P_{0}$ depends on an integration over all energies. Because the dielectric function is usually not known quantitatively over the entire range of energies, $P_{0}$ is difficult to evaluate. For interpretation of data it is best treated as a fit parameter. 
I now apply these ideas to angle-integrated PE, saving ARPES for later work. I assume that $P(\omega)$ is independent of emission angle, which should be true for the near-normal emissions typical for the incident photon energies used in most cases. The observed intensity $I(\omega, T)$, if electrons are emitted from a material with a temperature-independent density of states $N(\omega)$, is

$$
I(\omega, T)=P_{0}(T) N(\omega) f(\omega)+\int_{0}^{\infty} P\left(\omega-\omega^{\prime}, T\right) N\left(\omega^{\prime}\right) f\left(\omega^{\prime}\right) d \omega^{\prime}
$$

which must then be convoluted with an instrumental resolution function. The 'intrinsic' temperature dependence comes entirely from the Fermi function $f(\omega)$, but this dependence is very minor; I restrict the argument to relatively low $\mathrm{T}$.

I first consider a model system for illustrative purposes. For emission at a given $\vec{k}$ (ARPES), the observed intensity should consist of a main peak at $\omega=\epsilon_{\vec{k}}$ and an asymmetric tail below this, a rather common observation. For the angle-integrated quantity $I(\omega, T)$, we obtain a two-component result according to Eq. 6: the actual density of states $N(\omega)$ and a downshifted loss curve. Can this mimic a pseudogap ? Let $N(\omega)=N_{0}$ over some wide energy range $(\sim e V)$ below $\mu$, so that there is no actual pseudogap. Let the model system be a Drude conductor:

$$
\sigma(\omega)=\frac{\sigma_{0}}{1-i \omega \tau(T)}
$$

This expression is then substituted in Eq. 6 to produce Fig. 1 for two conductivities. The parameters for the dashed curve are: $\rho_{0}=1 / \sigma_{0}=110 \mu \Omega-\mathrm{cm}$ and $\tau=4 \times 10^{-14} \mathrm{~s}$. The parameters for the solid curve are: $\rho_{0}=1 / \sigma_{0}=44.5 \mathrm{~m} \Omega-\mathrm{cm}$ and $\tau=10^{-16} \mathrm{~s}$. Both curves have $P_{0}=0.01$ and $\mathrm{T}=38 \mathrm{~K}\left(\mathrm{k}_{B} \mathrm{~T}=3.3 \mathrm{meV}\right) . \sigma_{0} / \tau$ is held fixed in the figure. The point is very simple: in the Drude model $\sigma_{0} / \tau$ is just $n e^{2} / m^{*}$, where $n$ is the carrier concentration and $m^{*}$ is the effective mass, so that all of the temperature dependence in the conductivity occurs in the relaxation time, as in a conventional metal with no gap or pseudogap. The changes in the observed intensity arise entirely from extrinsic effects. The other parameters are held fixed as well. Note that these DC resistances are very high by the 
standards of ordinary metal physics, but quite typical of the CMR systems at temperatures comparable to or below the metal-insulator (M-I) transition. In the highly resistive state, the fields penetrate into the material and losses are high, whereas the loss is relatively low for the high conductivity state that screens the field. The plots have been normalized in the conventional manner by setting the intensities equal at a binding energy where they have leveled out - here at $-350 \mathrm{meV}$. The results are not very sensitive to this number. The popular midpoint method used to determine a "pseudogap" would give a value of about $50 \mathrm{meV}$. The dashed curve represents a system at the Mott conductivity - the borderline at which the loss effects become important. In good metals with $\rho_{0}<100 \mu \Omega$-cm losses become negligible, and the observed spectra reflect the actual density of states faithfully.

The curves demonstrate that in a system with a M-I transition, the observed intensity will change due to "extrinsic" effects. In general, there will be a motion of weight away from the Fermi energy as one approaches the insulating state. If such motion is observed in experiment it may not have anything to do with an actual pseudogap in the density of states.

Considering now actual spectra, angle-integrated $\mathrm{PE}$ on the CMR material $\mathrm{La}_{0.67} \mathrm{Ca}_{0.33} \mathrm{MnO}_{3}$ shows a number of unusual and striking features, represented by the points in Fig. 2, taken from Park et al. [1]. The material has a M-I transition at $260 \mathrm{~K}$. In the metallic state at $80 \mathrm{~K}$, there is a strong negative slope in $I(\omega)$ for at least $0.6 \mathrm{eV}$ below $\mu$. There is a sharp break in slope at $\mu$, presumably indicative of a nonzero density of states at $\mu$. In the insulating state at $280 \mathrm{~K}$ there appears to be no Fermi edge at all - the observed intensity is flat at $\mu$ and weight has moved back from $\mu$. There is even upward curvature in the data, as opposed to the downward curvature of the Fermi function. There is nothing in the usual theory of metals to account for any of these observations, and they certainly do not agree with band calculations [9]. These features have been taken to indicate a pseudogap [10], but they can be produced by extrinsic effects.

In Fig. 2, I plot the data points at two temperatures against the theory (Eq. 6). It is necessary to take a model for the frequency-dependent conductivity, which is not entirely 
Drude-like in the manganates. I have adopted a simplified version of the model of Okimoto el al. [11] in which there is a frequency-independent part $\sigma_{01}$ and a Drude part $\sigma_{d}(\omega)$ which is as in Eq. 7. This introduces an additional parameter $r=\sigma_{01} / \sigma_{d}(0)$ which measures the relative strength of the two components. The authors of Ref. [11] base their model on the analysis of their data on optical conductivity of $\mathrm{La}_{1-x} \mathrm{Sr}_{x} \mathrm{MnO}_{3}$ which is isoelectronic to the the calcium-doped system. Some sharp structure in the optical data, presumably due to phonons, is neglected in the model. If included, it might account for some of the additional small structure observed in PE.

The parameters for the upper curve are: $\tau=5 \times 10^{-14} \mathrm{~s}, \rho_{0}=1 / \sigma_{d}(0)=0.296 \mathrm{~m} \Omega-\mathrm{cm}$, $\mathrm{T}=80 \mathrm{~K}, P_{0}=0.0025$ and $r=0.2$. The parameters for the lower curve are: $\tau=10^{-14} \mathrm{~s}$, $\rho_{0}=1 / \sigma_{d}(0)=1.48 \mathrm{~m} \Omega$-cm, $\mathrm{T}=280 \mathrm{~K}, P_{0}=0$ and $r=0.25$. The curves are normalized to agree at a binding energy of $600 \mathrm{meV}$.

Again in this case, there is no change in the underlying density of states and the change in the theoretical intensity is entirely due to extrinsic effects.

To make a convincing case for a pseudogap from PE material a careful analysis of data using Eq. 6 is required to extract a pseudogap from experimental data. This suggests that meaningful investigation of electronic structure in poorly conducting materials requires a combination of PE with optical conductivity and electron energy loss measurements. This allows us to apply Eq. 6 and back out the density of states. A simple check can always be made. The inelastic part of the spectrum is inversely proportional to the speed of the outgoing electron, as may be seen from Eq. 1. Hence, to be genuine, a pseudogap must be present in the observed intensity at all incoming photon energies.

One may make some qualitative statements about the current situation in some of the more important classes of materials beyond the CMR manganates.

In good-quality high- $\mathrm{T}_{c}$ superconductors, the conductivity in the $a-b$ plane typically exceeds the Mott value. However, the conductivity along the $c$-axis is often less. Thus, these materials form a marginal case for the loss mechanism described here. There are other very strong indications that the pseudogap in the underdoped materials is quite real. ARPES 
itself shows that the pseudogap is momentum-dependent, which the loss is not. There is also corroboration from other experiments, tunneling being perhaps the most persuasive because it is also a direct measure of the DOS [12]. On the other hand, details of lineshapes may still be affected by extrinsic processes in high- $\mathrm{T}_{c}$ materials. A distinct sharpening of quasiparticle-like peaks is often observed as the temperature is lowered and the DC conductiviity increases, suggesting a decrease in energy loss.

In $1 \mathrm{D}$ systems, conductivity in two directions is very low, and one might expect the losses to be substantial. Intriguingly, it often appears to be the case that the gap or pseudogap measured in PE is greater than that given by other experiments. In $\left(\mathrm{TaSe}_{4}\right)_{2} \mathrm{I}$, for example, the PE gap at low temperatures is about $500 \mathrm{meV}$, whereas other experiments give values near $250 \mathrm{meV}$ [14. Another well-known example is TTF-TCNQ. At room temperature, DC transport data may be interpreted as that of a highly anisotropic gapless metal [13], but a pseudogap of $120 \mathrm{meV}$ is observed in ARPES [2]. These are only two of numerous examples of this puzzling mismatch which can be cited in 1D conductors. Such results are a strong indication that extrinsic processes are influencing the photoelectron spectrum in these systems. 


\section{REFERENCES}

[1] B. Dardel, D. Malterre, M. Grioni, P. Weibel, and Y Baer, Phys. Rev. Lett. 67, 3144 (1991); A. Terrasi, M. Marsi, H. Berger, G. Margaritondo, and M. Onellion, Phys. Rev. $B$ 52, $5592(1995)$.

[2] F. Zwick, D. Jérome, G. Margaritondo, M. Onellion, J. Voit, and M. Grioni, Phys. Rev. Lett. 81, 3144 (1998).

[3] A. G. Loeser et al., Science 273, 325 (1996); H. Ding et al. Nature (London) 382, 51 (1996).

[4] J.H. Park, C.T. Chen, S.-W. Cheong, W. Bao, G. Meigs, V. Chakarian, and Y.U. Izerda, Phys. Rev. Lett. 76, 4215 (1996).

[5] D. S. Dessau, T. Saitoh, C.-H. Park, Z.-X. Shen, Y. Moritomo and Y. Tokura, Intern. Journ. of Mod. Phys. B (to be published).

[6] M.R. Norman, M. Randeria, H. Ding, and J.C. Campuzano, to be published, cond-mat 9809206)

[7] H. Ibach, Surf. Sci. 299/300, 116 (1994) reviews this work.

[8] D.A. Shirley, Phys. Rev. B 5, 4709 (1972); S. Tougaard and B. Jørgensen, Surf. Sci. 143, 482 (1984).

[9] W.E. Pickett and P.J. Singh , Phys. Rev. B 53, 1146 (1996).

[10] D.S. Dessau and Z.-X. Shen, to be published in "Handbook of Magnetoresistive Oxides", ed. Y. Tokura.

[11] Y. Okimoto, T. Katsufuji, T. Arima, and Y. Tokura, Phys. Rev. B 554206 (1997).

[12] Ch. Renner, B. Revaz, J.-Y. Genoud, K. Kadowaki, and Ø. Fischer, Phys. Rev. Lett. 80, 149 (1998). 
[13] J.R. Cooper, Phys. Rev. B 19, 2404 (1979).

[14] N. Shannon and R. Joynt, J. Phys: Cond. Matt. 8, 10493 (1996); N. Shannon and R. Joynt, to be published (cond-mat/9806169).

I thank M. Rzchowski, F.J. Himpsel, M. Norman, M.B. Webb, N. Shannon, A.V. Chubukov, and L. Bruch for helpful discussions. Supported by the NSF under the Materials Theory Program, grant DMR-9704972 and under the Materials Research Science and Engineering Center Program, grant DMR-96-32527. 


\section{FIGURES}

FIG. 1. Calculated photoemission spectra from an idealized solid with a constant density of states. The curves differ only in the relaxation times: the dashed curve has $\tau=10^{-14} \mathrm{~s}$ whereas the solid curve has $\tau=4 \times 10^{-16} \mathrm{~s}$. The emitted electrons thereby lose different amounts of energy on exiting the solid.

FIG. 2. Calculated (lines) and observed (points) photoemission spectra for $\mathrm{La}_{0.67} \mathrm{Ca}_{0.33} \mathrm{MnO}_{3}$. Parameters of the fit are given in the text. The lineshapes, which do not resemble ordinary densities of states, are strongly affected by inelastic processes, which indeed dominate the lower curve. Points are taken from Ref. [4]. 


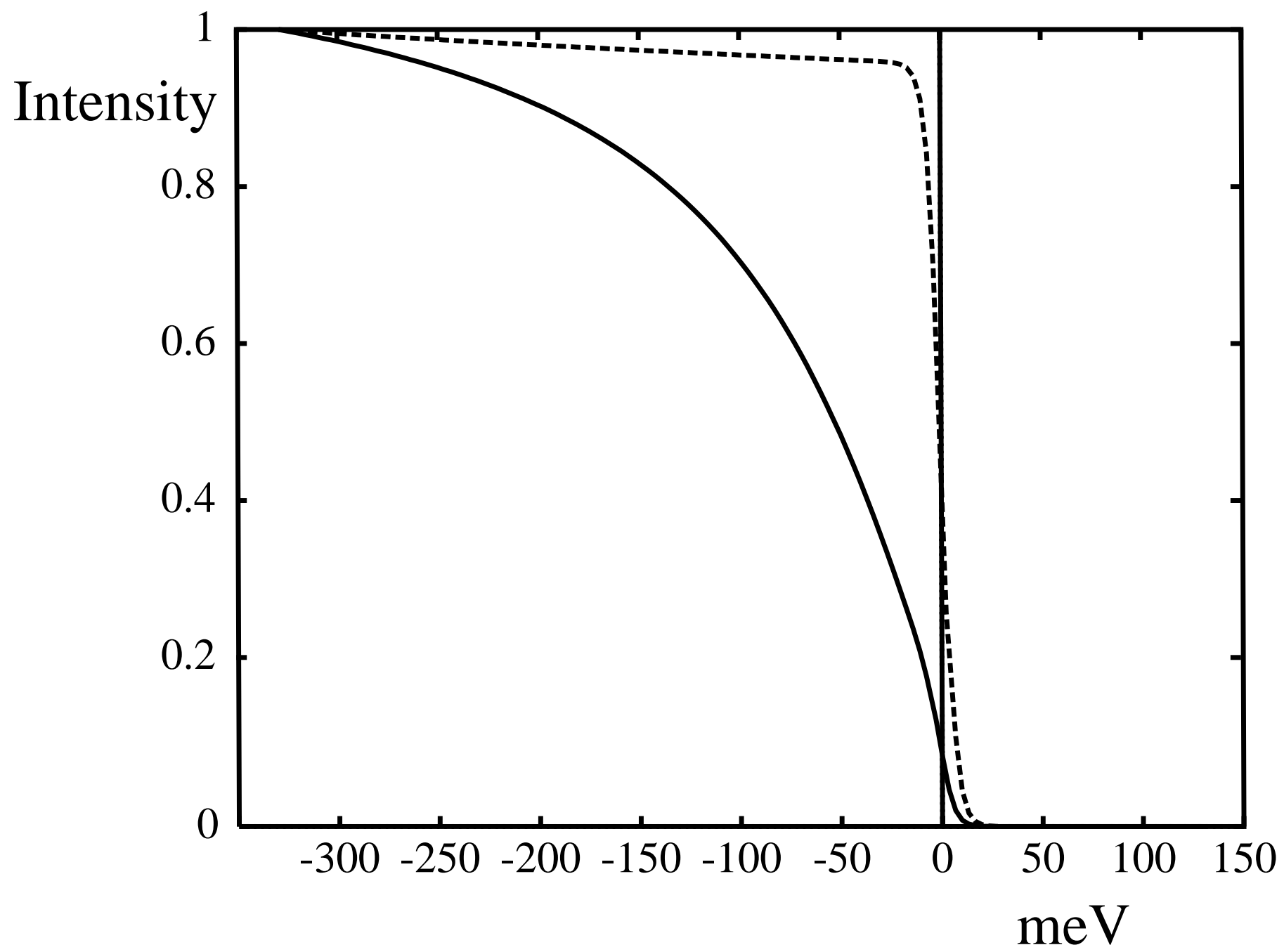

Fig. 1 


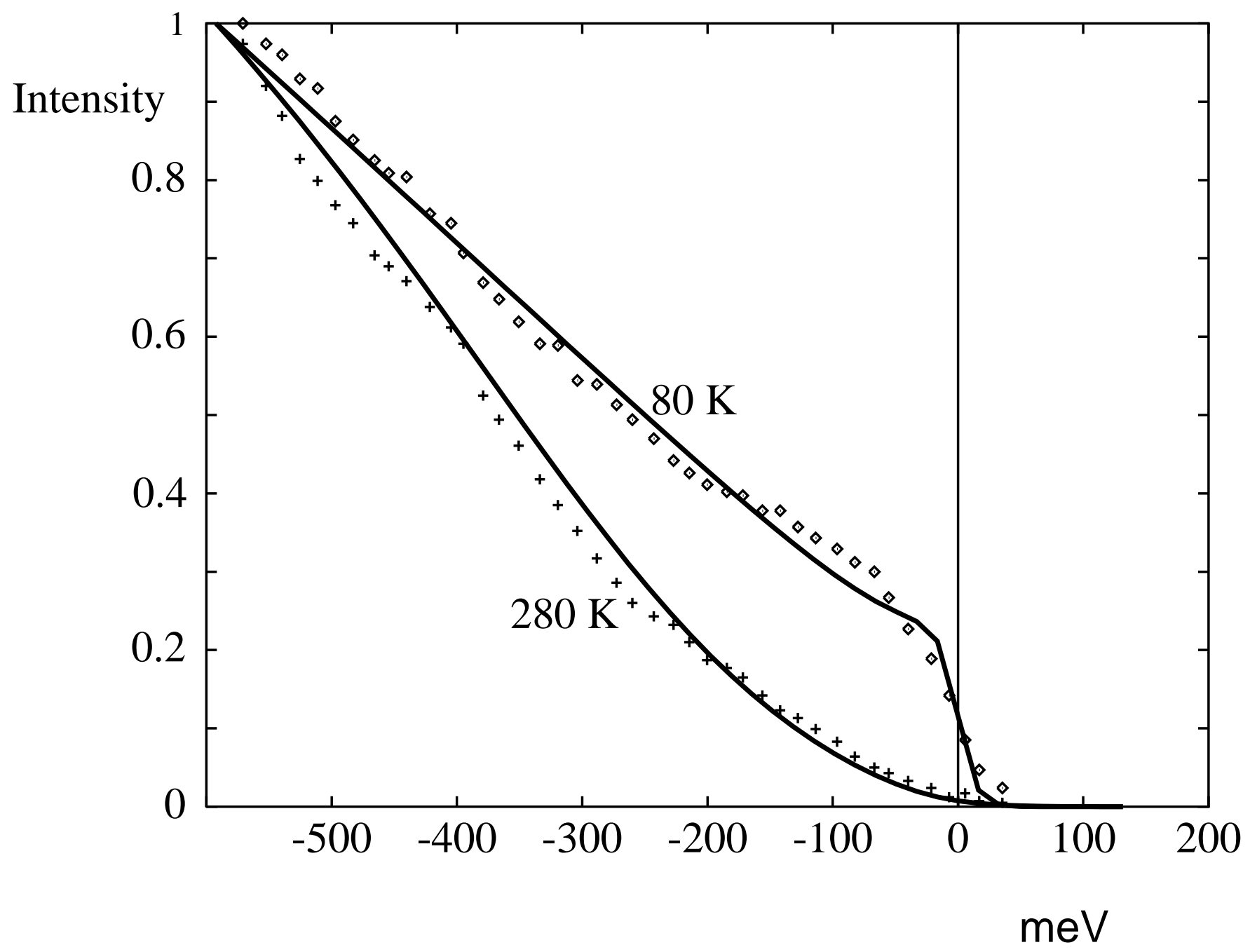

Fig. 2 